\title{
Overexposure analysis of pulsed distributed feedback laser source
}

\begin{abstract}
The goal of this study is to assess the risk of overexposure, when DFB dye laser is used for medical treatment in pulsed mode operation. Results of experimental study showing an unexpected rise of energy in pulses of distributed feedback dye laser (DFDL) output due to temperature phase gratings in dye cell during passively $\mathrm{Q}$ switched and mode-locked operation is reported. This unintended increase in the number of pulses, pulse duration, per pulse energy may cause side effects, when used for selective photothermolysis. To probe this phenomenon the most commonly used Rh6G dye was excited with 10 ï 20 pulses of second harmonic of a passively Q switched and mode-locked Nd:yttriumï aluminumï garnet(YAG) laser. The outputs of DFDL and Nd:YAG laser were recorded by an Imacon-675 streak camera. The peak of DFDL output pulses was found delayed proportionally from the peak of the Nd:YAG pulses by more than an inter-pulse period of excitation laser. A computer program was used to simulate the experimentally measured results to estimate the thermal decay constants and energy retained by medium. The delay between peaks of Nd:YAG (input) and DFDL (output) pulses was found to vary from 10 to 14 ns for various cavity lengths. It was interesting to note that for smaller inter-pulse periods the effect of gradual gain buildup satisfied the threshold conditions for some of the pulses that otherwise cannot lase. This may lead to unintended increase in energy fluence causing overexposure-induced bio effects.
\end{abstract}

Keyword: Risk assessment; Lasers; Medical applications 